\title{
Analytical Model of Adaptive CSMA-CA MAC For Reliable and Timely Clustered Wireless Multi-hop Communication
}

\author{
Raja Vara Prasad Y, Rajalakshmi Pachamuthu \\ Department of Electrical Engineering \\ Indian Institute of Technology Hyderabad \\ Hyderabad, India \\ Email: ee10p004,raji@iith.ac.in
}

\begin{abstract}
Reliability and delay of a single cluster wireless network is well analysed in the literature. Multi-hop communication over the number of clusters is essential to scale the network. Analytical model for reliability and end-to-end delay optimization for multi-hop clustered network is presented in this paper. Proposed model is a three dimensional markov chain. Three dimensions of markov model are the adaptable mac parameters of CSMA-CA. Model assumes wakeup rates for each cluster. Results show that reliability and delay are significantly improved than previous analytical models proposed. It has been observed that overall reliability of multi-hop link is improved, with reduction in endto-end delay is reduced even at lower wakeup rates of a cluster.
\end{abstract}

Keywords-clustered multi-hop network, Markov chain, Wakeup rates, end-to-end delay, reliability.

\section{INTRODUCTION}

The IEEE 802.15.4 standard has received considerable attention as a major low data rate and low power protocol for wireless sensor network (WSN) applications in industry, control, home automation, health care, and smart grids [1, 2] thereby enabling Internet of Things(IoT). Many of these applications require that packets are received with a given probability of success. In addition to such a reliability constraint, other applications ask for timely packet delivery [3]. It is known that IEEE 802.15.4 may have poor performance in terms of power consumption, reliability and delay [4], unless the medium access control (MAC) parameters are properly selected. Hence it follows that 1) it is essential to characterize the performance to understand the protocol limitations, and 2) it is instrumental to tune the IEEE 802.15.4 parameters to enhance the network lifetime and improve the quality of the service experienced by the applications running on top of the network.

This paper focuses on modelling and optimization of the performance metrics like reliability and delay of IEEE 802.15.4 for clustered multi-hop networks. Existing analytical studies of IEEE 802.15.4 are not adequate to capture the real world protocol behaviour, where there are retry limits to send packets, acknowledgement (ACK), minimum exponents and maximum backoffs.
The remainder of this paper is as follows. In Section 2, we summarize existing work for the analytical model and adaptive tuning of IEEE 802.15.4. Section 3 presents the system model based on a generalized Markov chain model of CSMA-CA with retry limits and unsaturated traffic regime. The analytical model results and adaptiveness to MAC parameters is discussed in Setion 4. Finally, Section 5 concludes the paper.

\section{RELATED WORK}

The modelling of IEEE 802.15.4 is similar to IEEE 802.11 [5] as CSMA-CA of both MAC are almost same. Both IEEE 802.15.4 and 802.11 MACs uses a binary exponential backoff scheme. Bianchis model describes the basic functionalities of the IEEE 802.11 with a Markov chain under ideal channel and saturated traffic conditions [6]. This model have been extended and used to analyse the packet reception rate in [7], delay in [8], the MAC layer service time and throughput in [9] and [10] respectively of IEEE 802.11. The analysis of IEEE 802.15.4 WSNs packet delay, throughput, and power consumption has been the focus of several simulations-based studies in [11] and [12], and recent analytical works also in [4],[13-16]. A Markov model for IEEE 802.15.4 based on Bianchis work, with ACK mechanism is discussed in [4] and [13]. Another Markov model with retransmissions and finite retry limits proposed in [15] to avoid collisions in slotted CSMACA. However, under unsaturated traffic with finite retry limits the power consumption and throughput expressions are far from coinciding with simulation results. In [16] the superframe structure, $\mathrm{ACK}$, and retransmissions are considered. However, Markov chain in [16] does not model the length of data and ACK packets, which is crucial low data rate IEEE 802.15.4 networks.

Wireless sensor networks are generally used for monitoring applications where sensors data is forwarded to the sink. Eventhough it is mentioned in the literature that sensor networks can be used in actuation systems only few application exploited in real time implementation. This 


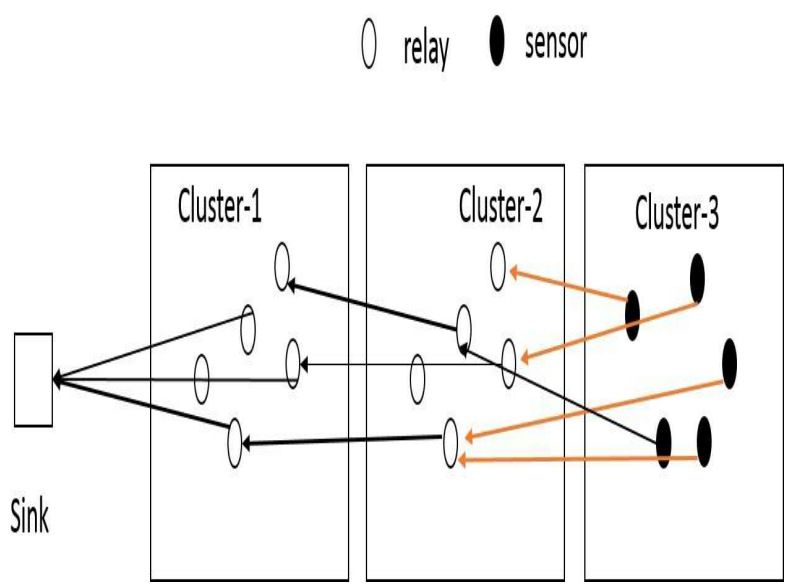

Figure 1. Proposed system model

is due to fact that existing 802.15.4 protocol stack don't guarantee the QoS requirements for the applications. A lot of research communities [17-18] have tried to meet the requirements like reliability, end-to-end delay for the monitoring applications. Some of the proposed protocols focuses on wireless system which can guarantee reliable and timely data [21] for the star networked systems. Analytical model proposed in [23] is based on clustered multi-hop network with wake up rates. Even though markov model in [23] has detailed analysis on reliability, delay and energy parameters, markov model has been proposed without ack mechanism and retry for collision. In this paper a markov chain model with possible mac parameters like minimum backoff exponent $\left(m_{0}\right)$ and maximum backoffs $(\mathrm{m})$ is proposed. Reliability, delay are analysed with adaptiveness in the $m_{0}$, and $\mathrm{m}$.

\section{SySTEM MODEL}

Wireless sensor network is based on 802.15.4 standard. Many researchers tried to model IEEEE 802.15.4 MAC [17-20] but a complete markov chain based MAC model is proposed in [21]. The model presented in [21] has most of the MAC parameters like minBE $\left(m_{0}\right)$, maxBackoffs $(\mathrm{m}), \max$ retries (n) etc. The analysis in [21] is for star topology where $\mathrm{n}$ nodes of a total $\mathrm{N}$ contend for the channel. We propose a wireless Cyber Physical System (CPS) with a centralized sink/controller and distributed actuation system. Model proposed in this paper is a clustered network where each cluster has sensors and relay nodes as shown in the figure-1. Sensor nodes are in the Edge cluster-3 and relay nodes are distributed between sensor and sink nodes as shown in the figure-1. Sensor nodes in edge cluster cannot send packets directly. Sensor nodes forward data to the nearest relay cluster as shown in the figure-1. Sensors data is forwarded to sink through multi-hop communication.
Model proposed has cluster topology with adaptive wake-up rates and sleep schedules to save the energy of battery operated nodes. A 3 dimensional markov chain model similar to [21] is developed to meet the requirements of reliable and timely communications. A detailed analytical model is presented in the form of equations as below.

A three dimensional markov chain based mathematical model is used to derive the required reliability and delay of CSMA-CA algorithm from one cluster to neighbor cluster. Three dimensions of the markov chain are $\mathrm{s}(\mathrm{t}), \mathrm{c}(\mathrm{t})$ and $\mathrm{r}(\mathrm{t})$ where $s(t)$ is stochastic processes representing the backoff stage $\mathrm{c}(\mathrm{t})$ is the state of the backoff counter and $\mathrm{r}(\mathrm{t})$ is state of retransmission counter at time $\mathrm{t}$ experienced by a node to transmit a packet, as shown in figure-3.

States $Q_{0}$ to $Q_{\mathrm{L}-1}$ consider the idle state when the packet queue is empty and the node is waiting for new packet arrivals. Markov chain shown in the figure3 has backoff windows with mac parameters like minimum backoff exponent, maximum backoff exponents, macMaxCSMABackoffs and maximum retries. Finally $(\mathrm{m}, \mathrm{k}, \mathrm{n})$ are dimensions of the markov chain. Main assumption of the markov chain is any node can start sensing in any random slot which results in independent probability for channel sensing probability $(\tau)$. Reliability and delay expressions are derived based on the markov chain discussed. All expressions are interms of $\alpha, \beta$ and $\tau$ where $\alpha$ is the probability of channel busy in CCA1 state, $\beta$ is the probability of channel busy in CCA2 state and $\tau$ is the probability that any node senses a channel. Finally expressions are converted into markov state transition probabilities as shown in the equation-3 to equation- 6 .

Analytical model presented below is for cluster topology, where in each cluster a node traverses different states like sleep, idle listen, active Tx of CSMA CA are as shown in the figure-2. Other states like sleep, awake are embedded into model with wake-up rates.

Equation-1 gives the probability of being channel busy in either CCA1 or CCA2.

$$
x=\alpha+(1-\alpha) * \beta
$$

where

$\alpha$ is the probability of channel busy in CCA1 state

$\beta$ is the probability of channel busy in CCA2 state

$\tau$ is the probability any node senses a channel

Equation-2 gives the probability of node taking $\mathrm{n}$ retries when it fails to transmit packet due to collision.

$$
\widehat{y}=1-(1-\tau)^{(\mathrm{n}-1)} *\left(1-x^{2}\right)
$$

Equation-3 to 6 are simplification terms in the main derivation of single cluster csma-ca reliability.

$$
r_{1}=(1+2 * x) *(1+\widehat{y})
$$




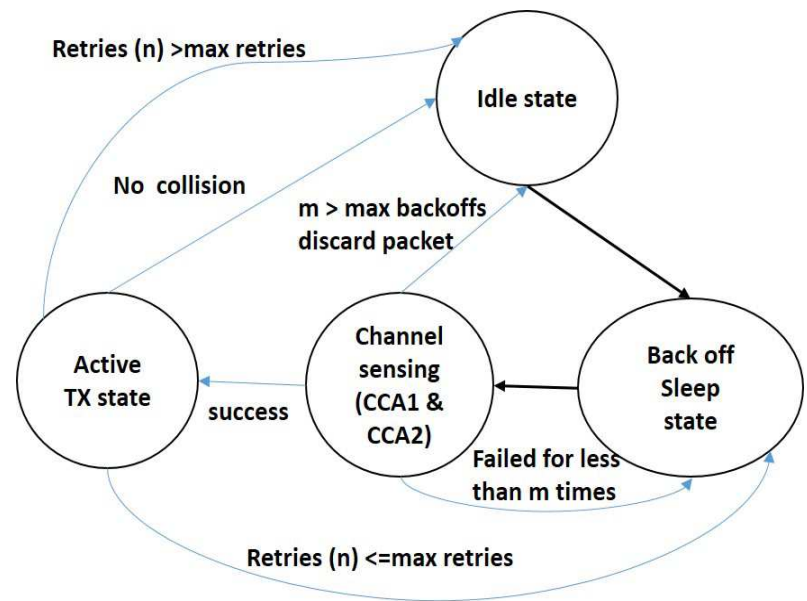

Figure 2. State diagram of a CSMA-CA states

$$
\begin{gathered}
k_{0}=\left(L_{0} * q\right) /(1-q) \\
r_{2}=L_{s} *\left(1-x^{2}\right)\left(1+\widehat{y}+k_{0}\left(1+\widehat{y}^{2}+\widehat{y}^{\mathrm{n}+1}\right)\right. \\
b_{0,0,0}=2 /\left(W_{0} * r_{1}+2 * r_{2}\right)
\end{gathered}
$$

Equation-7 and 8 are approximated $\tau$ and y to simplify the complex non-linear expressions without losing more than $5 \%$ accuracy.

$$
\begin{gathered}
\widetilde{\tau}=(1+x) *(1+\widehat{y}) * b_{0,0,0} \\
\widetilde{y}=\left(1-(1-\widetilde{\tau})^{(\mathrm{n}-1)}\right) *\left(1-x^{2}\right)
\end{gathered}
$$

finally equation-9 gives reliability of single cluster for csmaca.

$$
R_{1 \text {-cluster }}=1-x^{(\mathrm{m}+1)} *(1+\widetilde{y})-\widetilde{y}^{(\mathrm{n}+1)}
$$

Proposed model is for clustered multi-hop communication where there are $\mathrm{h}$ number of clusters. Even though we say clusters but there is no cluster head. Data from one cluster is forwarded to next cluster by random selection of node. A exponential wake up rate is assumed for a cluster to wake up. Nodes will forward data to forwarding region after it observes a beacon from forwarding region. Equation-10 gives a wake up probability equation in a cluster.

$$
\begin{gathered}
\psi_{\mathrm{sb}}(n)=\exp \left(-\mu_{c} *(n-1) / \lambda\right)-\exp \left(-\mu_{c} *(n) / \lambda\right) \\
\psi_{\mathrm{sb}}(1)=1-\exp \left(-\mu_{c} / \lambda\right)
\end{gathered}
$$

Finally reliability of data over a $\mathrm{h}$ number of clusters is given in equation-11 with $P_{\min }$ is minimum probability of worst channel condition in clusters.

$$
R_{\text {total }}=\left(\psi_{\mathrm{sb}} * P_{\min } * R_{1 \text {-cluster }}\right)^{h}
$$

Mean end-to-end delay for multi-hop clustered communication is in the equations-23. equation-12 to equation-17 are supporting equations to get delay of CSMA-CA of equation-22

$$
\begin{gathered}
S b=32 * 10^{-} 5 \\
b l=\max (\alpha,(1-\alpha) * \beta) \\
t_{1}=(1-b l) /\left(1-b l^{(\mathrm{m}+1)}\right) \\
T s=L+L a c k+1 \\
T s=L+L a c k+1 \\
P_{c}=\left(1-(1-\tau)^{(\mathrm{n}+1)}\right) \\
y=P_{c} *\left(1-x^{(\mathrm{m}+1)}\right)
\end{gathered}
$$

$$
t_{2}=\left(2 * W 0 *\left(1-(2 * b l)^{(\mathrm{m}+1)}\right)\right) /(1-2 * b l)
$$

$$
t_{3}=\left(3 *(m+1) * b l^{(\mathrm{m}+1)) /(1-\mathrm{bl})}\right.
$$

where

$\mathrm{Sb}$ is the unit backoff time

$P_{c}$ is the packet collision probability

$\mathrm{y}$ is the probability of a node entering in next retry state W0 is the minimum backoff window

Ts is transmission time

Tc is packet collision time

$D_{\text {avg }}$ is the approximated backoff delay

$D_{\mathrm{avg}}=2 * S b *\left(1+0.25\left(t_{1} *\left(t_{2}-t_{3}\right)+3 * b l /(1-b l)-(W 0+1)\right)\right)$

$$
\begin{aligned}
D_{\mathrm{csma}}=T s+\text { Delay }_{\mathrm{avg}}+ & \left(y /(1-y)-\left((n+1) *\left(y^{(\mathrm{n}+1)}\right)\right)\right. \\
& \left./\left(1-y^{(\mathrm{n}+1)}\right)\right) *\left(T s+\text { Delay }_{\mathrm{avg}}\right)
\end{aligned}
$$

Delay of overall link from sensor to sink is sum of CSMA-CA delay and cluster wake up delay

$$
D_{\text {mean }}=\text { Delay }_{\mathrm{csma}} * h+h / \mu_{c}
$$




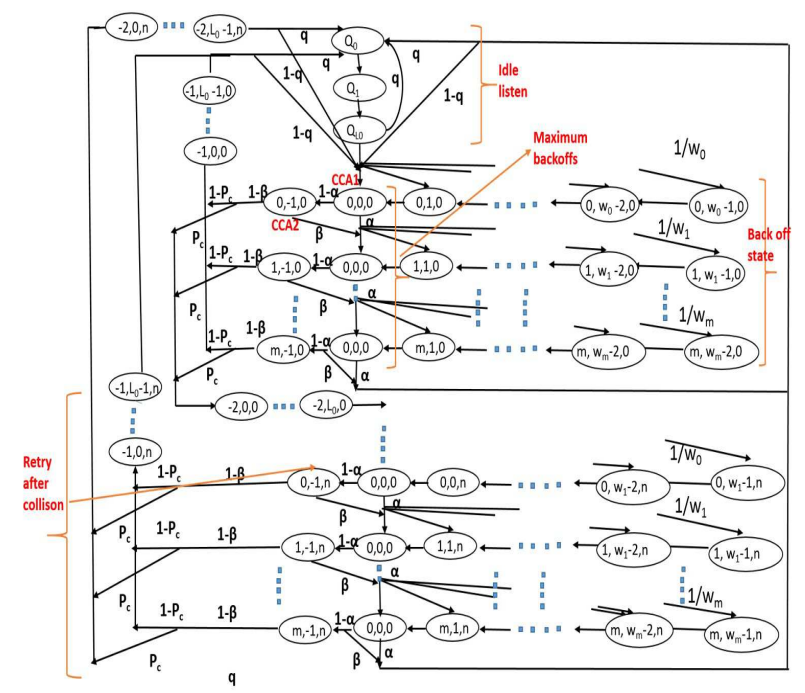

Figure 3. 3 dimensional markov chain model of a IEEE 802.15.4 standard

\section{Analytical Results}

Analytical expressions for reliability and end-to-end delay derived in system model section is simulated in Matlab to analyse the effect of unidirectional data flow in multi-hop clustered communication. Reliability of link are analysed compared to existing single wake-up strategy in [22]. Even though there are $\mathrm{n}$ nodes the expressions in equation- 10 is approximated upto $n=2$ as this approximation will not effect accuracy more than $3 \%$. Results for reliability are as shown in the figure-4. Figure-4 plot is Reliability versus wake up rate. Reliability of the proposed 3 dimension markov model is better than 2 dimension Breath model as shown in the figure-4. Reliability of the proposed model is almost $40 \%$ more than existing breath during lower wakeup rates. Higher reliability at lower wake up rates improves the duration of the network. Adapting to the mac parameters like maximum backoffs (m) still improves the reliability by $20 \%$ compared to that without adaptive maximum backoffs $(m)$ as shown in the figure-5. This is due to the fact that instead of fixing a maximum backoffs for a given wakeup rate and packet rate, if maximum backoffs can be set to optimum value reliability is improved. Similarly other mac parameters adaptability also improves the reliability.

Delay of the proposed model is also compared with breath. Delay of the proposed model is mainly due to CSMA-CA and cluster wakeup rates. Delay due to cluster wakeup is not part of this papers work. Optimal cluster wake up rates is well studied in [23]. Figure-6 plots end-to end delay versus wake up rate of a cluster. Delay of the proposed model is approximately $25 \%$ less than Breath model. Delay due to CSMA-CA is analysed with optimally selecting MAC parameters. Delay of the proposed model is better than

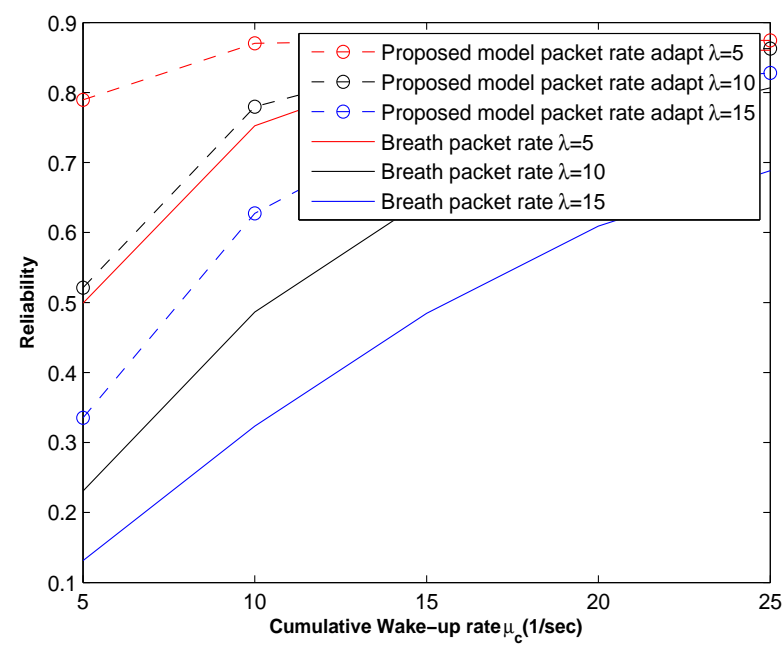

Figure 4. Comparison of proposed and Breath analytical results for reliability

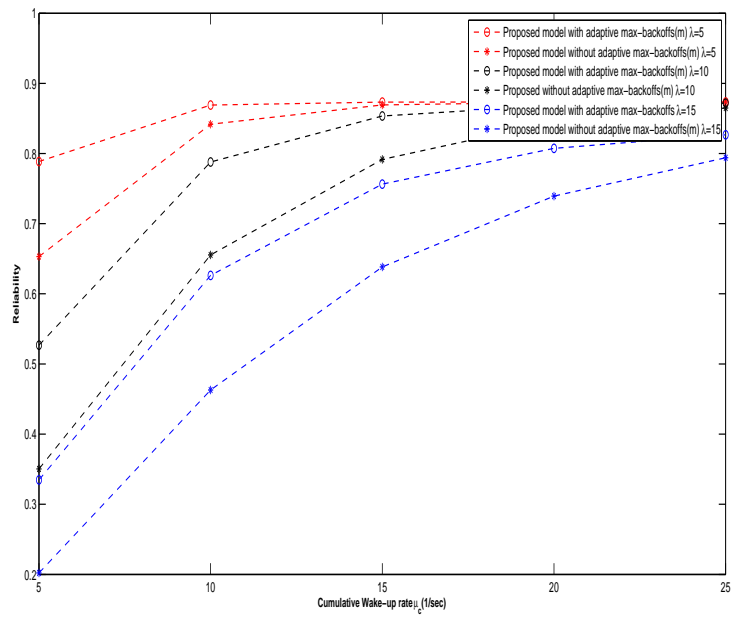

Figure 5. Comparison of analytical results for reliability with and without Adaptive max backoffs

Breath model as shown in the figure- 6 . Adapting the mac parameters to reduce delay is shown in the figure-7, but the improvement due to adaptation at lower wake up rates is less than $2 \%$ due to the fact that major part of the delay is due to cluster wakeup rate compared to delay incurred by CSMA-CA. Adaptiveness of MAC will be effective when the delay bounds are in tens of milliseconds.

\section{CONCLUSION}

Analytical model for a uni directional clustered data flow is developed using a 3 dimensional markov chain. Each cluster is assigned a wake up rate for energy optimization. Reliability and end-to-end delay of the overall 


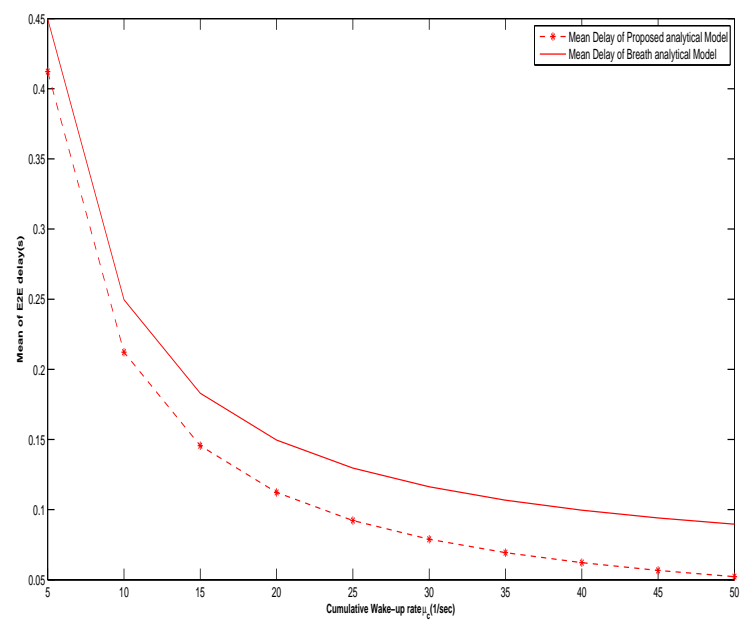

Figure 6. Comparison of proposed and Breath analytical results for mean end-to-end delay

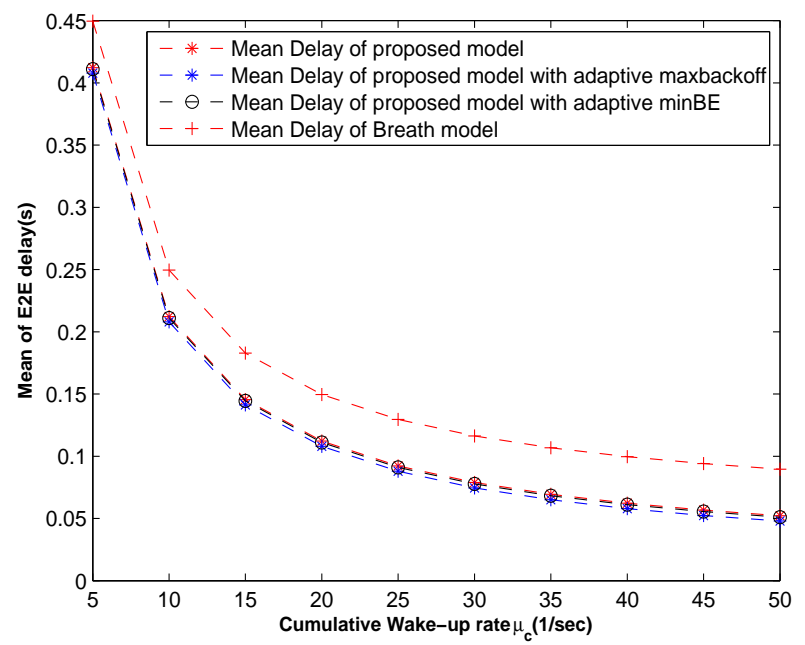

Figure 7. Comparison of analytical results for mean end-to-end delay with and without Adaptive mac parameters $\left(m_{0}\right.$ and $\left.\mathrm{m}\right)$

unidirectional link (sensors to sink) is derived from the proposed model. Reliability and end-to-end delay of the proposed model is compared with existing 2 dimensional markov Breath model. Results show that reliability is significantly higher than breath model even at lower wake up rates. Adaptability to the Mac parameter max backoff still improve the reliability. End-to-end delay is also analysed for the proposed model but the reduction in the delay is less due to higher delay of cluster wake up rate. Analysis of simultaneous mac parameters adaption with energy as main objective function is future scope of research.

\section{REFERENCES}

[1] IEEE Std 802.15.4-2996, September, Part 15.4: Wireless Medium Access Control (MAC) and Physical Layer (PHY) Specifications for Low-Rate Wireless Personal Area Networks (WPANs), IEEE, 2006. [Online]. Available: http://www.ieee802.org/15

[2] A. Willig, K. Matheus, and A. Wolisz "Wireless technology in industrial networks", Proceedings of the IEEE, 2005.

[3] T. Abdelzaher, T. He, and J. Stankovic,'Feedback control of data aggregation in sensor networks,", in IEEE CDC, December 2004 ..

[4] S. Pollin, M. Ergen, S. C. Ergen, B. Bougard, F. Catthoor, A. Bahai, and P. Varaiya, "Performance analysis of slotted carrier sense IEEE 802.15.4 acknowledged uplink transmissions", in IEEE WCNC, 2008..

[5] IEEE Std 802.11 Wireless LAN Medium Access Control (MAC) and Physical Layer (PHY) Specifications, IEEE, 1999. [Online]. http://www.ieee802.org/11.

[6] G. Bianchi, "Performance analysis of the IEEE 802.11 distributed cordination function,", IEEE Journal on Selected Areas in Communications, vol. 18, March 2000..

[7] P. Chatzimisios, A. C. Boucouvalas, and V. Vitsas,'IEEE 802.11 packet delay a finite retry limit analysis,", in IEEE GLOBECOM, 2003

[8] Z. Hadzi-Velkov and B. Spasenovski,"Saturation throughputdelay analysis of IEEE 802.11 in fading channel," in IEEE ICC, 2003.

[9] O. Tickioo and B. Sikdar, "Queueing analysis and delay mitigation in IEEE 802.11 random access MAC based wireless networks," in IEEE INFOCOM, 2004.

[10] H. Wu, Y. Peng, K. Long, S. Cheng, and J. Ma, "Performance of reliable transport protocol over IEEE 802.11 wireless LAN: Analysis and enhancement," in IEEE INFOCOM, 2002, pp. 599607.

[11] J. Zheng and M. L. Lee,"A comprehensive performance study of IEEE 802.15.4,", in IEEE Press Book, 2004.

[12] A. Koubaa, M. Alves, and E. Tovar, "A comprehensive simulation study of slotted CSMA-CA for IEEE 802.15.4 wireless sensor networks,", in IEEE IWFCS, Jun 2006, pp. 183192.

[13] S. Pollin, M. Ergen, S. C. Ergen, B. Bougard, L. V. D. Perre, F. Catthoor, I. Moerman, A. Bahai, and P. Varaiya, "Performance analysis of slotted carrier sense IEEE 802.15.4 medium access layer,", in IEEE GLOBECOM, 2006, pp. 16.

[14] J. Misio, S. Shaf, and V. Misio, "Performance of a beacon enabled IEEE 802.15.4 cluster with downlink and uplink traffic,", IEEE Trans. Parallel and Distributed Systems, pp. 361376, 2006

[15] P. K. Sahoo and J. P. Sheu, "Modeling IEEE 802.15.4 based wireless sensor network with packet retry limits,", in ACM PEWASUN, 2008. 
[16] C. Y. Jung, H. Y. Hwang, D. K. Sung, and G. U. Hwang, "Enhanced markov chain model and throughput analysis of the slotted CSMA-CA for IEEE 802.15.4 under unsaturated traffic conditions,", IEEE Transactions on Vehicular Technology, vol. 58, no. 1, pp. 473478, 2009.

[17] M. Buettner, G. Yee, E. Anderson, and R. Han, "X-MAC: A short preamble MAC protocol for duty-cycled wireless sensor networks,", in ACM SenSys, 2006..

[18] F. Stann and J. Heidemann, "RMST: Reliable Data Transport in Sensor Networks,"”, in IEEE SNPA, 2003.

[19] S. Kim, R. Fonseca, P. Dutta, A. Tavakoli, D. Culler, P. Levis, S. Shenker, "Flush: a reliable bulk transport protocol for multihop wireless networks,", in ACM SenSys, 2007.

[20] O. B. Akan and F. Akyildiz, and I. Stoica, "Event-to-sink reliable transport in wireless sensor networks,", IEEE Transactions on Networking, vol. 13, no. 5, pp. 1003 1016, 2005.

[21] P. Park, P. Di Marco, C. Fischione, and K. H. Johansson, "Adaptive IEEE 802.15.4 Protocol for Reliable and Timely Communications,", IEEE/ACM Transactions on Networking, 2009

[22] C. Fischione, P. Park, S. C. Ergen, K. H. Johansson, and A. Sangiovanni- Vincentelli, "Analytical Modeling and Optimization of Duty-cycles in Preamblebased IEEE 802.15.4 Wireless Sensor Networks,", IEEE/ACM Transactions on Networking, 2009

[23] P. Park, C. Fischione, A. Bonivento, K. H. Johansson and A. Sangiovanni-Vincentelli, "Breath: an Adaptive Protocol for Industrial Control Applications using Wireless Sensor Networks", Mobile Computing, IEEE Transactions, June 2011 Volume:10 ,Issue: 6 